\title{
GLOSSÁRIO DE TERMOS USADOS NO PLANEJAMENTO DE FÁRMACOS (RECOMENDAÇÕES DA IUPAC PARA 1997)
}

\author{
Carlos Mauricio R. Sant'Anna*
}

Departamento de Química, Universidade Federal Rural do Rio de Janeiro, 23851-970 Seropédica - RJ; Departamento de Fármacos, Universidade Federal do Rio de Janeiro, 21949-910 Rio de Janeiro - RJ

Recebido em 13/12/01

O presente glossário é uma tradução para o português (aprovada pelo Comitê Brasileiro para Assuntos de Química junto à IUPAC) do artigo Glossary of Terms Used in Computational Drug Design publicado em Pure Appl. Chem. 1997, 69, 1137, preparado por $H$. van de WaterBeemd, M. S. Tute, R. E. Carter, G. Grassy, H. Kubinyi, Y. C. Martin e P. Willet.

\section{INTRODUÇÃO}

O planejamento computacional de fármacos é uma área de rápido crescimento que atualmente é um componente muito importante da disciplina de química medicinal. Ao mesmo tempo, muitos químicos medicinais carecem de treinamento formal significativo nesta área e podem não ter uma compreensão clara da terminologia usada, precisando entretanto entender conceitos, acompanhar resultados de pesquisa, definir problemas para o planejamento computacional de fármacos e utilizar seus resultados.

Neste contexto, o Comitê da Seção de Química Medicinal da IUPAC sentiu que seria útil desenvolver um glossário de termos usados no planejamento computacional de fármacos para fins de referência fácil. Também há a possibilidade de que em países diferentes certos termos possam não ter o mesmo significado e, em tais casos, seria valioso tentar estabelecer um padrão de definição internacional. Deste modo, um Grupo de Trabalho de sete especialistas na área foi organizado, o qual construiu um glossário de cerca de 100 termos. Definições concisas mas suficientemente explanatórias foram formuladas, baseadas em uma variedade de fontes da literatura e referências chaves fornecidas. ${ }^{a}$

Recomendações 1997;@ 1997 IUPAC. Reimpresso de Pure Appl. Chem. 1997, 69, 1137

\section{REGISTROS ALFABÉTICOS}

Algumas das definições também aparecem no Glossary of Terms used in Medicinal Chemistry (recomendações da IUPAC 1997; (C) 1997 IUPAC). Estas definições estão marcadas com um asterisco.
Para algumas definições, a forma mais estendida tomada do Glossary of Terms in Theoretical Organic Chemistry é incluída em fonte menor.

\section{GLOSSÁRIO DE TERMOS USADOS NO PLANEJAMENTO COMPUTACIONAL DE FÁRMACOS}

Abordagem Extratermodinâmica - A abordagem extratermodinâmica envolve a correlação entre variáveis que, do ponto de vista estritamente termodinâmico, não são relacionadas. Esta é a base da análise de Hansch usada em QSAR tradicional, ou clássica ${ }^{1}$. (veja Análise de Hansch)

Algoritmo Genético - Um algoritmo genético é um algoritmo de otimização baseado nos mecanismos da evolução Darwiniana. O algoritmo usa procedimentos de mutação aleatória, cruzamento e seleção para gerar modelos ou soluções melhores a partir de uma população ou amostra de partida originalmente aleatória ${ }^{2}$.

AMBER - AMBER (do inglês Assisted Model Building with Energy Refinement, Construção Assistida de Modelos com Refinamento de Energia) é um programa de mecânica molecular bastante conhecido para cálculos de proteínas e de ácidos nucléicos. ${ }^{b}$ (veja Mecânica molecular)

Análise Comparativa de Campos Moleculares (CoMFA*, do inglês Comparative Molecular Field Analysis) - A análise comparativa de campos moleculares (CoMFA) é um método de QSAR-3D que usa técnicas estatísticas de correlação para a análise de relações quantitativas entre a atividade biológica de um conjunto de compostos com um alinhamento específico e suas propriedades eletrônicas e estéricas tridimensionais ${ }^{\mathrm{c}}$. Outras propriedades, tais como hidrofobia e ligação hidrogênio, também podem ser incorporadas na análi$\mathrm{se}^{3,4}$. (veja QSAR-3D, hidrofobia). ${ }^{\mathrm{d}}$

Análise Conformacional - A análise conformacional consiste na exploração dos arranjos espaciais (formas) energeticamente favoráveis de uma molécula (conformações). Na análise utiliza-se mecânica molecular, dinâmica molecular, cálculos químico-quânticos ou análise de dados estruturais determinados experimentalmente por RMN ou cristalografia de raios-X, por exemplo. Os métodos de

*e-mail: santanna@ufrrj.br

${ }^{a}$ N.T.: Por se tratar de um campo de evolução muito rápida, muitos termos novos surgiram desde a publicação do glossário em língua inglesa e a atual tradução. Este trabalho deve ser considerado como um esforço inicial para suprir as necessidades de padronização dos termos do planejamento computacional de fármacos na língua portuguesa, os quais já são usados com frequiência em artigos de química medicinal publicados em nosso país.

${ }^{\mathrm{b}}$ N.T.: AMBER também refere-se ao campo de força. Apesar deste campo de força ter sido parametrizado e definido para proteínas e ácidos nucléicos, parâmetros têm sido adicionados para estendê-lo para cálculos de outras classes de moléculas.

${ }^{\mathrm{c}}$ N.T.: Na maior parte dos casos as relações quantitativas envolvem atividades farmacológicas, mas o método pode ser aplicado igualmente a conjuntos de moléculas planejadas para causar qualquer ação sobre organismos vivos, inclusive tóxica, como é o caso dos pesticidas.

${ }^{\mathrm{d} N}$.T.: O método CoMFA também pode ser usado para a análise de relações quantitativas entre afinidades (por receptores, etc.) com propriedades físicoquímicas tridimensionais. 
mecânica molecular e químico-quânticos são empregados para computar as energias conformacionais, enquanto que os métodos de busca sistemática e aleatória, de Monte Carlo, de dinâmica molecular e de geometria de distâncias (freqüentemente combinados com procedimentos de minimização de energia) são usados para explorar o espaço conformacional. (veja Geometria de distâncias, Dinâmica molecular, Mecânica molecular, Técnica de Monte Carlo e Cálculos químico-quânticos)

Análise de Discriminantes - Análise de discriminantes é uma técnica estatística usada para encontrar um conjunto de descritores que podem ser usados para detetar e racionalizar a separação entre classes de atividades.

Análise de Free-Wilson ( $F W$ - A análise de Free-Wilson é uma técnica de regressão que usa a presença ou ausência de substituintes ou grupos como os únicos descritores moleculares em correlações com a atividade biológica ${ }^{1}{ }^{\mathrm{e}}$

Análise de Hansch* - A análise de Hansch é a investigação da relação quantitativa entre a atividade biológica de uma série de compostos e seus parâmetros físico-químicos relativos aos substituintes ou à molécula toda que representam os efeitos hidrofóbicos, eletrônicos, estéricos e outros, usando a metodologia de correlação de regressão múltipla ${ }^{1,5}{ }^{\mathrm{f}}$

Análise de Componentes Principais (PCA, do inglês Principal Components Analysis) - Análise de componentes principais é um método de redução de dados que usa técnicas matemáticas para identificar padrões em uma matriz de dados. O principal elemento deste método consiste na construção de um pequeno conjunto de novas variáveis ortogonais, ou seja, não correlacionadas, derivadas de uma combinação linear das variáveis originais.

Análise por Agrupamento - Análise por agrupamento consiste no agrupamento de grandes conjuntos de dados (por exemplo, conjuntos de dados químicos ou farmacológicos) com base em critérios de similaridade para variáveis apropriadamente escaladas, que representam os dados de interesse. Critérios de similaridade (baseados em distância, associativos, correlativos e probabilísticos) entre vários grupos facilitam o reconhecimento de modelos e revelam estruturas de outra forma ocultas ${ }^{6-8}$.

Análise Populacional de Mulliken - Análise populacional de Mulliken é um método para distribuir os elétrons em átomos com a finalidade de gerar cargas atômicas parciais. Os resultados são fortemente dependentes do conjunto de bases usado.

Análise populacional de Mulliken - Um esquema de partição, baseado no uso das matrizes de densidade e de recobrimento, para distribuir os elétrons de uma entidade molecular de algum modo fracionário entre suas várias partes (átomos, ligações, orbitais). Como em outros esquemas usados para distribuir a densidade eletrônica em moléculas, a análise populacional de Mulliken é arbitrária e fortemente dependente do conjunto de bases empregado. Contudo, a comparação de análises populacionais para uma série de moléculas é útil para uma descrição quantitativa das interações intramoleculares, de reatividade química e de regularidades estruturais.

Análise por Regressão - Análise por regressão é o uso de métodos estatísticos para modelar um conjunto de variáveis dependentes, Y, em termos de combinações de variáveis independentes, X. A análise inclui métodos como a regressão linear múltipla (MLR, do inglês multiple linear regression) e mínimos quadrados parciais (PLS, do inglês partial least squares).

Arquivo $\mathrm{Mol}$ - Um arquivo mol é uma tabela que contém tipos de átomos, conectividade e informação 2D ou 3D mais ou menos arbitrária sobre uma molécula. Formatos de arquivo bem conhecidos incluem o arquivo MOL usado pela MDL Information Systems Inc. (por exemplo, a base de dados MACCS), o arquivo MOL2 usado pela Tripos Associates (por exemplo, no pacote de modelagem SYBYL) ou o formato CSSR.

Arquivo PDB - Um arquivo PDB (do inglês Protein Data Bank, Banco de Dados de Proteínas) é um arquivo ASCII (do inglês American Symbolic Code for Information Interexchange = text, Código Simbólico Americano para Troca de Informação) usado para armazenar as coordenadas atômicas de uma molécula, geralmente uma proteína ou ácido nucléico. (veja $P D B$ )

Árvore de Decisão de Topliss* - Árvore de decisão de Topliss é um esquema operacional para o planejamento de análogos9.

Busca Conformacionalmente Flexível (CFS, do inglês Conformationally Flexible Searching) - Busca conformacionalmente flexível é uma pesquisa de bancos de dados de estruturas tridimensionais que leva em conta a flexibilidade das moléculas.

Busca em Base de Dados Tridimensional - Busca em base de dados tridimensional é uma técnica para encontrar protótipos que usa estruturas tridimensionais de compostos armazenadas em uma base de dados.

Cálculos ab initio - Cálculos $a b$ initio são cálculos mecânicoquânticos que usam equações exatas, sem aproximações, que envolvem a população eletrônica total da molécula.

Métodos mecânico-quânticos $a b$ initio (sinônimo com métodos mecânico-quânticos não empíricos) - Métodos de cálculos mecânico-quânticos independentes de qualquer experimento que não seja a determinação de constantes fundamentais. Os métodos são baseados no uso da equação de Schrödinger completa para tratar todos os elétrons de um sistema químico. Na prática, aproximações são necessárias para restringir a complexidade da função de onda eletrônica e tornar seu cálculo possível.

Cálculos AM1 - Cálculos AM1 são cálculos semi-empíricos de orbital molecular desenvolvidos na Universidade de Austin no Texas (AM1 = Austin Model 1, Modelo Austin 1). Estes cálculos envolvem os elétrons de valência dos átomos da molécula. Eles são um desenvolvimento dos cálculos $\mathrm{MNDO}^{10}$. (veja cálculos $\left.M N D O\right)$

Cálculos CNDO/2 - Cálculos CNDO/2 são cálculos semi-empíricos de orbital molecular $(M O)$ que usam a negligência completa do recobrimento diferencial.g (veja cálculos de orbital molecular (MO))

Cálculos de Hückel Estendidos - Cálculos de Hückel estendidos são cálculos de orbital molecular (MO) semi-empíricos de nível baixo de aproximações.

Método de Hückel estendido - Um método mecânico-quântico semi-empírico com todos os elétrons de valência que usa as mesmas aproximações, menos a aproximação $\mathrm{p}$ e a negligência das integrais de recobrimento, da teoria do orbital molecular de Hückel. $\mathrm{O}$ método reproduz relativamente bem as formas e a ordem dos níveis de energia dos orbitais moleculares. A consideração do recobrimento torna possível a desestabilização líquida causada pela interação de dois orbitais duplamente ocupados.

Cálculos de Orbital Molecular (MO, do inglês Molecular Orbital) - Cálculos de orbital molecular (MO) são cálculos mecâni-

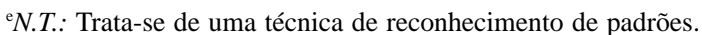

${ }^{\mathrm{f}}$ N.T.: Também conhecida como Análise de Hansch e Fujita. Estas relações de energia livre, desenvolvidas por análise de regressão, não são termodinâmica clássica, mas sim extratermodinâmica (veja Abordagem Extratermodinâmica).

${ }^{\mathrm{g}}$ N.T.: o termo refere-se ao recobrimento das funções de base de átomos diferentes (as funções de base do mesmo átomo não se sobrepõem por serem ortogonais). 
co-quânticos baseados na equação de Schrödinger, que podem ser subdivididos em métodos semi-empíricos e ab initio. (veja Cálculos ab initio)

Teoria do orbital molecular - Um método da mecânica quântica molecular que usa funções de um elétron (orbitais) como uma aproximação para a função de onda total.

Cálculos de Perturbação de Energia Livre - Cálculos de perturbação de energia livre são procedimentos matemáticos usados em estudos de dinâmica molecular para converter gradualmente uma espécie química em outra em um ciclo termodinâmico.

Cálculos MINDO/3 - Cálculos MINDO/3 (do inglês Modified Intermediate Neglect of Differential Overlap, Negligência Intermediária Modificada do Recobrimento Diferencial) são cálculos de orbital molecular (MO) semi-empíricos ${ }^{11}$.

Cálculos MM2 - Cálculos MM2 envolvem cálculos de mecânica molecular que usam a versão 2 do programa de campo de força $M M 2^{12}$, amplamente distribuído.

Cálculos MNDO - Cálculos MNDO são cálculos semi-empíricos de orbital molecular (MO), que usam como aproximação uma negligência modificada de recobrimento diatômico (diferencial).

Cálculos PCILO - Cálculos PCILO (do inglês Perturbative Configuration Interaction using Localized Orbitals, Interação de Configuração Perturbativa usando Orbitais Localizados) são cálculos semi-empíricos de orbital molecular relacionados com cálculos CNDO/2 e MNDO.

Cálculos Mecânico-quânticos - Cálculos mecânico-quânticos são cálculos de propriedades moleculares baseados na equação de Schrödinger que levam em conta as interações entre os elétrons em uma molécula.

Cálculos PM3 - PM3 (do inglês Parametric Method 3) é um programa de cálculo semi-empírico de orbital molecular largamente usado (veja Métodos semi-empíricos). ${ }^{\mathrm{h}}$

Campo de Força - O campo de força é um conjunto de funções e parametrização usadas em cálculos de mecânica molecular.

Campo de Força - Dentro da abordagem da mecânica molecular, um conjunto de funções de potencial que definem as energias de estiramento de ligação e de distorção de ângulo de ligação (tanto de valência quanto diedro) de uma molécula, quando comparadas com a sua conformação não tensionada (aquela caracterizada pelos valores padrões de comprimentos e de ângulos de ligação). Um conjunto de constantes de força empíricas transferíveis é previamente atribuído e a aproximação harmônica é geralmente empregada. Alguns campos de força podem conter termos de interação entre átomos não ligados, de efeitos eletrostáticos, de ligação hidrogênio e de outros efeitos estruturais, assim como considerar efeitos de anarmonicidade. Na espectroscopia vibracional, é resolvido o problema inverso da determinação de um conjunto de constantes de força e de outros parâmetros de funções de energia potencial selecionadas que coincidiria com freqüências vibracionais experimentalmente observadas de uma determinada série de moléculas congêneres.

Campo e Potencial Eletrostáticos - O campo e o potencial eletrostáticos são propriedades de uma molécula que surgem da interação entre uma sonda carregada, tal como uma carga pontual unitária positiva representando um próton, e a molécula alvo. Este campo e o potencial são usados em estudos de relações quantitativas estrutura-atividade tridimensionais (3D-QSAR) e para comparar ou estimar a semelhança de um conjunto de moléculas.

Potencial eletrostático - uma propriedade física igual em magnitude à energia eletrostática entre a distribuição de carga estática, r(r), de um sistema atômico ou molecular e uma carga pontual unitária positiva localizada em r. O potencial eletrostático $\mathrm{V}(\mathrm{r})$ que é produzido em qualquer ponto $r$ pelos elétrons e pelos núcleos (A) de um sistema é dado por:

$$
V(r)=\sum \frac{Z_{A}}{\left|R_{A}-r\right|}-\int \frac{r\left(r^{\prime}\right) d r^{\prime}}{\left|r^{\prime}-r\right|} .
$$

Combinação Linear de Orbitais Atômicos (LCAO, do inglês Linear Combination of Atomic Orbitals) - A combinação linear de orbitais atômicos é um método matemático usado em cálculos mecânico-quânticos. Este método expressa a aproximação da função do orbital molecular como sendo uma combinação linear de orbitais atômicos escolhidos como funções de base.

Conjunto de Bases - Um conjunto de bases é um conjunto de funções matemáticas usadas em cálculos de orbital molecular (MO, do inglês Molecular Orbital), por exemplo, o conjunto de bases 631G* usado em cálculos ab initio. 6-31G* e expressões similares referem-se ao tipo de função matemática usada. ${ }^{\text {i }}$ (veja Cálculos de Orbital Molecular (MO))

Conjunto de bases - Um conjunto de funções de base empregado para a representação de orbitais moleculares. Pode-se distinguir o conjunto mínimo de bases (inclui uma função de base para cada orbital atômico ocupado de SCF (do inglês Self-Consistent Field, Campo Autocoerente) com números quânticos principal e de momento angular distintos); conjunto de bases de valência divididas (inclui dois ou mais tamanhos de funções de base para cada orbital de valência); conjunto de bases duplo zeta (DZ) (um conjunto de bases de valência divididas que inclui exatamente o dobro das funções do conjunto mínimo de bases); conjunto de bases estendido (o conjunto maior do que o conjunto de bases duplo zeta); conjunto de bases polarizadas (incorpora funções de base de número quântico angular mais alto, além daquele requerido pelo átomo no estado fundamental; permite aos orbitais mudar não apenas o tamanho, mas também a forma); conjunto de bases com funções difusas e outros.

Conjunto de Bases STO-3G - Um conjunto de bases STO-3G é um conjunto de orbitais do tipo Gaussiana (GTO, do inglês Gaussiantype orbital), cada um dos quais usa três funções Gaussianas para aproximar um orbital do tipo Slater (STO, do inglês Slater-type orbital). Conjuntos de bases modernos mais estendidos incluem STO3-21G ou STO-KG.

Constante $\sigma$ de Hammett - A constante de Hammett é um descritor eletrônico de substituinte que reflete as propriedades doadoras ou receptoras de elétrons de um substituinte ${ }^{13}$.

Constante $\pi$ de Hansch-Fujita - A constante $\pi$ de Hansch-Fujita descreve a contribuição de um substituinte para a lipofilia de um composto ${ }^{5}$.

${ }^{\mathrm{h} N . T .:}$ Nesta tradução. A definição traduzida do original, incorreta, diz que "PM3 é um programa de mecânica molecular semi-empírico largamente usado (veja Mecânica molecular)".

${ }^{i} N$. T.: Quase todos os cálculos ab initio modernos utilizam conjuntos de bases com orbitais representados por funções de probabilidade de Gauss. Estas funções podem ser subdivididas em orbitais internos, compactos, e externos, mais difusos. O termo 6-31G, por exemplo, significa que os orbitais do "caroço" consistem de 6 funções Gaussianas e os da camada externa estão subdivididos em um conjunto de $3+1$ funções Gaussianas. A introdução de orbitais $d$ no cálculo é indicada por um asterisco. Dois asteriscos indicam que um conjunto de orbitais $p$ está presente para os átomos de hidrogênio. Os cálculos do tipo Møller-Plesset (MP2, MP4, etc.) incluem correções para a correlação eletrônica. 
Constante Fragmentária Lipofílica ( $f$ ou $f^{\prime}$ ) - A constante fragmentária lipofílica de um substituinte ou fragmento molecular representa a contribuição do substituinte ou fragmento molecular para a lipofilia ${ }^{14-16}{ }^{\mathrm{j}}$

Correlações Estrutura-Propriedade (SPC*, do inglês StructureProperty Correlation) - Correlações estrutura-propriedade referemse a todos os métodos estatísticos matemáticos usados para correlacionar qualquer propriedade molecular (intrínseca, química ou biológica) a qualquer outra propriedade, usando técnicas de regressão estatística ou de reconhecimento de padrões ${ }^{17}$.

CSSR - O formato de arquivo CSSR (do inglês Crystal Structure Search Retrieval, Recuperação de Pesquisa de Estrutura Cristalina) é um dos vários formatos usados pelo Banco de Dados de Estruturas Cristalinas de Cambridge (CSD, do inglês Cambridge Structural Database) para armazenar estruturas moleculares. Este formato é usado em muitos pacotes de programas de modelagem molecular.

Descritores Moleculares - Descritores moleculares são termos que caracterizam um aspecto específico de uma molécula ${ }^{18}$.

Diagrama de Craig - O diagrama de Craig é um diagrama de dois parâmetros de substituintes (por exemplo, valores $\pi$ de HanschFujita e $\sigma$ de Hammett) usado no planejamento de análogos. ${ }^{k}$

Dinâmica Molecular - Dinâmica molecular é um procedimento de simulação que consiste na computação do movimento dos átomos em uma molécula ou de átomos individuais ou moléculas em sólidos, líquidos e gases, de acordo com as leis de movimento de Newton. As forças que agem nos átomos, necessárias para simular os seus movimentos, são calculados usando campos de força de mecânica molecular. ' (veja Mecânica molecular)

(Dis)similaridade Molecular - (Dis)similaridade molecular é um número para expressar a relação estrutural entre pares de moléculas, por exemplo, os chamados coeficientes de Carbo, de Hodgkin ou de Tanimoto $^{19,20}$.

Energia do Orbital Molecular Desocupado de Menor Energia (LUMO, do inglês Lowest Unoccupied Molecular Orbital) - A energia do orbital molecular desocupado de menor energia é obtida por cálculos de orbital molecular e representa a afinidade eletrônica de uma molécula ou a sua reatividade como eletrófilo. (veja Energia do Orbital Molecular Ocupado de Maior Energia (HOMO))

Energia do Orbital Molecular Ocupado de Maior Energia (HOMO, do inglês Highest Occupied Molecular Orbital) - A energia do orbital molecular ocupado de maior energia é obtida por cálculos de orbital molecular e se relaciona com o potencial de ionização de uma molécula e com sua reatividade como nucleófilo. (veja Energia do Orbital Molecular Desocupado de Menor Energia (LUMO))

Orbital de fronteira - Os orbitais moleculares que envolvem o orbital molecular ocupado de maior energia (HOMO, do inglês Highest Occupied Molecular Orbital) e o orbital molecular desocupado de menor energia (LUMO, do inglês Lowest Unoccupied Molecular Orbital) de uma dada entidade. No caso de uma entidade molecular com um número ímpar de elétrons, quando o seu HOMO é ocupado por um único elétron, tal orbital molecular é denominado orbital molecular unicamente ocupado (SOMO, do inglês Singly Occupied Molecular Orbital). Dependendo das propriedades de seu parceiro reativo, o SOMO de uma dada espécie pode funcionar como HOMO ou como LUMO. A importância especial dos orbitais de fronteira é devida ao fato de que uma grande variedade de reações químicas ocorre em uma posição e em uma direção onde a sobreposição do HOMO e do LUMO dos respectivos reagentes é máxima.

Espaço de Parâmetros - $\mathrm{O}$ espaço de parâmetros é um espaço multidimensional compreendido pelos descritores em um conjunto de dados.

Estatística Multivariada - Estatística multivariada é um conjunto de ferramentas estatísticas para analisar matrizes de dados (por exemplo, químicos e biológicos), que usam técnicas de regressão ou de reconhecimento de padrões.

Estudos de Ancoramento - estudos de ancoramento são técnicas computacionais para a exploração dos possíveis modos de interação de um substrato a um dado receptor, à uma enzima ou a outro sítio de ligação.

Forma Molecular - A forma molecular é um atributo de uma molécula que trata da extensão espacial, forma, estrutura ou geometria. É descrita freqüentemente pelos eixos principais, ovalidade ou índices de conectividade, por exemplo.

Formato de Arquivo - $\mathrm{O}$ formato de arquivo (molecular) descreve o arranjo de um arquivo de dados de computador. É um conjunto de instruções sobre como uma molécula é codificada com relação à sua conectividade, aos tipos de átomos e às coordenadas, podendo também conter dados bibliográficos.

Formato de Arquivo $M O L$ - O formato de arquivo MOL é usado para codificar estruturas, subestruturas e conformações químicas como tabelas de conecção baseadas em texto. É usada pela $M D L$ Information Systems Inc. (por exemplo, nos seus programas MACCS e ISIS $)^{21}$.

Geometria de Distâncias - Geometria de distâncias é um método matemático usado para construir modelos moleculares tridimensionais $(3 D)$ a partir de um conjunto de distâncias interatômicas aproximadas (por exemplo, experimentos de efeito nuclear Overhauser (NOE, do inglês nuclear Overhauser effect) em ressonância magnética nuclear $(R M N)$ sugerem apenas faixas de distâncias). A geometria de distâncias pode ser usada para definir um farmacóforo 3D a partir de um conjunto de moléculas que têm o mesmo mecanismo de ação ou para gerar geometrias semelhantes de complexos ligantereceptor usando restrições de distância intermolecular ${ }^{22}$.

Geração de Farmacóforo - Geração de farmacóforo é um procedimento para extrair as mais importantes características estruturais comuns que sejam relevantes para uma dada atividade biológica de uma série de moléculas com mecanismo de ação semelhante.

GOLPE (do inglês Generating optimal linear PLS estimations) - Gerando estimativas PLS lineares ótimas. É uma técnica avançada de mínimos quadrados parciais (PLS, do inglês Partial least squares) para seleção de variáveis, usada em estudos de relações quantitativas estrutura-atividade tridimensionais (3D-QSAR) para manusear conjuntos de dados muito grandes. (veja Mínimos quadrados parciais $(P L S))$

Gráficos Moleculares* - Gráficos moleculares são técnicas para a visualização e a manipulação de moléculas em um sistema de apresentação gráfica.

GRID - GRID é um programa para o mapeamento receptor/ ligante. O programa calcula as energias de interação entre sondas e moléculas alvo em pontos de interação em uma grade $3 \mathrm{D}^{23}$.

${ }^{\mathrm{j} N . T .:}$ Nesta tradução. Originalmente definido como "Constante Fragmentária Hidrofóbica" (Hydrophobic Fragmental Constant).

${ }^{\mathrm{k}} N$. T.: Também existe o diagrama de Craig 3D.

${ }^{1}$ N.T.: O cálculo de trajetórias pode ser realizado com métodos semi-empíricos, e mesmo com métodos ab initio (no caso de eventos com tempo de vida curto). Em geral, estes cálculos são referidos na literatura com termos específicos, como DRC (do inglês Dynamic Reaction Coordinate, Coordenada de Reação Dinâmica), "Semiempirical Direct Dynamics" (Dinâmica Direta Semi-empírica) e "Ab Initio Direct Dynamics" (Dinâmica Direta Ab Initio).

${ }^{\mathrm{m} N}$ N.T.: Os termos de Carbo são termos de similaridade química. 
Hamiltoniano - O Hamiltoniano é uma função matemática operador usada em cálculos de orbitais moleculares ${ }^{10}$.

Hidrofilia $^{* n}$ - Hidrofilia é a tendência de uma molécula em ser solvatada por água.

Hidrofobia* ${ }^{*}$ - Hidrofobia é a associação de grupamentos ou moléculas apolares em um ambiente aquoso que se origina da tendência da água excluir moléculas apolares ${ }^{24-26}$.

Índice de Conectividade Molecular - Um índice de conectividae molecular é um descritor numérico de topologia molecular ${ }^{27}$.

Índice Topológico - Um índice topológico é um valor numérico associado à constituição química para a correlação da estrutura química com várias propriedades físicas, reatividade química ou atividade biológica.

Índice Topológico - A base numérica para os índices topológicos é fornecida (dependendo de como um gráfico molecular é convertido em um valor numérico) ou pela matriz de adjacências ou pela matriz de distâncias topológicas. Nesta última, a distância topológica entre dois vértices é o número de arestas no caminho mais curto entre estes.

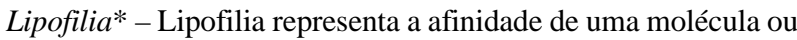
de um fragmento por um ambiente lipofílico. É comumente medida por seu comportamento de distribuição em um sistema bifásico, seja líquido-líquido (por exemplo, coeficiente de partição em 1-octanol/ água) ou sólido-líquido (retenção em sistema de cromatografia líquida de alta eficiência com fase reversa ( $R P-H P L C$, do inglês reversed-phase high-performance liquid chromatography) ou de cromatografia de camada delgada ( $T L C$, do inglês thin-layer chromatography)).

Mapeamento de Receptor* - Mapeamento de receptor é uma técnica usada para descrever as características geométricas ou eletrônicas de um sítio de ligação quando não estão disponíveis dados estruturais suficientes para este receptor ou enzima. Geralmente, a cavidade do sítio ativo é definida comparando-se a sobreposição de moléculas ativas com a de moléculas inativas.

Mecânica Molecular - Mecânica Molecular é o cálculo das geometrias e energias conformacionais de moléculas usando uma combinação de campos de força empíricos ${ }^{28}$.

Mecânica molecular (sinônimo de método de campo de força) Método de cálculo das características geométricas e de energia de entidades moleculares baseado em funções de potencial empíricos (veja campo de força), cuja forma é tomada da mecânica clássica. O método pressupõe que as funções de potencial possam ser transferidas dentro de um conjunto de moléculas semelhantes. Uma suposição é feita sobre os comprimentos e ângulos "naturais" de ligação, cujos desvios resultam em tensão de ligação e tensão angular, respectivamente. As forças de Van der Waals e eletrostáticas repulsivas e atrativas entre átomos não ligados também são levadas em conta.

Método do Átomo Unido - O método do átomo unido é uma simplificação usada por programas de mecânica molecular tais como AMBER e CHARMM. A influência de grupos de átomos ou fragmentos moleculares é aproximada tratando-os como um único átomo.

Método Seqüencial Simplex - O método seqüencial simplex é um método de planejamento experimental usado para a otimização rápida de propriedades.

Métodos Semi-Empíricos - Métodos semi-empíricos são cálculos de orbital molecular que usam vários graus de aproximação e que usam apenas elétrons de valência.

Métodos mecânico-quânticos semi-empíricos - Os métodos que usam parâmetros derivados de dados experimentais para simplifi- car os cálculos computacionais. A simplificação pode ocorrer em vários níveis: simplificação do Hamiltoniano (por exemplo, como no método de Hückel estendido), avaliação aproximada de certas integrais moleculares (veja, por exemplo, recobrimento diferencial zero), simplificação da função de onda (por exemplo, o uso da aproximação do elétron p como no método Pariser-Parr-Pople).

Minimização de Energia - Minimização de energia é um procedimento matemático para localizar as conformações estáveis (mínimos de energia) de uma molécula, determinadas por cálculos de mecânica molecular ou mecânico-quânticos. (veja Mecânica molecular, Cálculos mecânico-quânticos)

Mínimos Quadrados Parciais (PLS, do inglês Partial Least Squares) - A projeção para estruturas latentes por mínimos quadrados parciais é um método robusto de regressão multivariada generalizada que usa projeções para resumir um grande número de variáveis potencialmente colineares. ${ }^{29}$

Modelagem Molecular - Modelagem molecular é a investigação das estruturas e das propriedades moleculares usando a química computacional e as técnicas de visualização gráfica visando fornecer uma representação tridimensional, sob um dado conjunto de circunstâncias.

Modelo de Homologia - Um modelo de homologia é um modelo de uma proteína, cuja estrutura tridimensional é desconhecida, construído a partir de dados de coordenadas de raios-X de proteínas semelhantes ou usando técnicas de alinhamento e argumentos de homologia, por exemplo.

Orbitais Atômicos (AO, do inglês Atomic Orbitals) - Orbitais atômicos são funções matemáticas (por exemplo, funções Gaussianas ou de Slater) usadas em cálculos mecânico-quânticos. Um conjunto de orbitais atômicos descritos por uma determinada função é o conjunto de bases dos orbitais atômicos. (veja orbitais tipo Slater).

Orbital (Atômico ou Molecular) - Uma função de onda que depende explicitamente das coordenadas espaciais de apenas um elétron.

Orbitais do Tipo Gaussiana (GTO, do inglês Gaussian-Type Orbital) - Orbitais do tipo Gaussiana são funções matemáticas usadas em cálculos ab initio. Eles substituíram os orbitais do tipo Slater por causa da maior eficiência computacional resultante. (veja orbitais do tipo Slater)

Orbitais do Tipo Slater (STO, do inglês Slater-Type Orbital) Orbitais do tipo Slater são funções matemáticas que envolvem funções exponenciais, usadas em cálculos mecânico-quânticos $a b$ initio. Estas funções simulam a distribuição eletrônica em átomos e foram usadas em cálculos $a b$ initio, mas atualmente foram substituídas por orbitais do tipo Gaussiana (veja orbitais do tipo Gaussiana)

Orbital atômico do tipo Slater (STO) - A função exponencial sobre um átomo; sua dependência radial é dada por $\mathrm{Nr}^{\mathrm{n}-1} \exp (-\zeta \mathrm{r})$, onde $\mathrm{n}$ é o número quântico principal e $\zeta$ é o expoente do orbital (constante de blindagem) derivado de considerações empíricas. A dependência angular é geralmente introduzida multiplicando-se a radial por um harmônico esférico $\mathrm{Y}_{\mathrm{lm}}(\theta, \phi)$.

Parâmetro Estérico de Taft (Es) - O parâmetro estérico de Taft é um parâmetro de reação relativo que codifica o retardamento da velocidade de reação devido ao tamanho de um grupo substituinte.

Parâmetros de Swain-Lupton (F e R) - Os parâmetros de SwainLupton $(F$ e $R$ ) são descritores de campo eletrônico e de ressonância derivados das constantes de Hammett ${ }^{15}$.

Parâmetros STERIMOL de Verloop - Os parâmetros STERIMOL definidos por Verloop são um conjunto de parâmetros de comprimento e de largura do substituinte ${ }^{30}$. 
$P D B$ - O banco de dados de proteínas (PDB, do inglês Protein Data Bank) mantido na Laboratório Nacional Brookhaven, Upton, New York, que contém estruturas de raios-X de várias centenas de proteínas. (veja Arquivo $P D B)^{\mathrm{p}}$

Planejamento Baseado em Estrutura* - Planejamento baseado em estrutura é uma estratégia para novas entidades químicas baseada na estrutura tridimensional $(3 D)$ de um alvo obtida de estudos de raios-X ou de ressonância magnética nuclear, ou de modelos de homologia de proteínas.

Planejamento de Fármacos - O planejamento de fármacos inclui não apenas o planejamento do ligante, mas também a farmacocinética e a toxidez, que estão na maior parte das vezes além das possibilidades do planejamento auxiliado pela estrutura ou por computador. Todavia, ferramentas quimiométricas apropriadas, que incluem planejamento experimental e estatística multivariada, podem ser valiosas no planejamento e avaliação de experimentos e resultados farmacocinéticos e toxicológicos. O termo planejamento de fármacos é mais frequientemente usado do que o termo correto "Planejamento de Ligantes".

Planejamento de Fármacos Auxiliado por Computador (CADD*, do inglês Computer-Assisted Drug Design) - O planejamento de fármacos auxiliado por computador envolve todas as técnicas com o auxílio do computador usadas para descobrir, planejar e otimizar compostos biologicamente ativos com uso suposto de fármacos. (veja Planejamento de fármacos)

Planejamento de Ligantes - É o planejamento de ligantes que usa informações estruturais sobre o alvo ao qual os ligantes devem se ligar, freqüentemente tentando maximizar a energia de interação. (veja Estudos de ancoramento)

Planejamento De Novo* - Planejamento de novo é o planejamento de compostos bioativos pela construção em incrementos de um modelo de ligante dentro do sítio ativo do receptor ou enzima, cuja estrutura é conhecida de dados de raios-X ou de ressonância magnética nuclear (RMN).

Planejamento D-ótimo - Planejamento D-ótimo é uma técnica de planejamento experimental baseada na otimização do determinante calculado a partir da matriz de variância-covariância dos descritores. É usado para maximizar a eficiência do planejamento fatorial fracionário (incompleto). (veja Planejamento fatorial, Planejamento fatorial fracionário).

Planejamento Experimental - Planejamento experimental é o uso de métodos matemáticos e estatísticos para selecionar o número mínimo de experimentos ou de compostos para a varredura ótima do espaço de descritores ou variáveis.

Planejamento Fatorial (FD, do inglês Factorial Design) - Planejamento Fatorial é uma técnica de planejamento experimental na qual cada variável (fator ou descritor) é investigada em níveis fixos. No FD de dois níveis, cada variável pode assumir dois valores, por exemplo, lipofilias alta e baixa.

Planejamento Fatorial Fracionário (FDD, do inglês Fractional Factorial Design) - O planejamento fatorial fracionário é uma técnica de planejamento experimental que usa um fator de redução de forma a limitar o número de experimentos a um número mais baixo do que o obtido pelo planejamento fatorial.

Planejamento Molecular - Planejamento molecular é a aplicação de todas as técnicas que levam à descoberta de novas entidades químicas com propriedades específicas necessárias para a aplicação pretendida.
Planejamento Molecular Auxiliado por Computador (CAMD*, do inglês Computer-Assisted Molecular Design) - O planejamento molecular auxiliado por computador é a investigação das estruturas e propriedades moleculares usando a química computacional e técnicas de visualização gráfica.

Potenciais de Interação Molecular (MIP, do inglês Molecular Interaction Potential) - Potenciais de interação molecular (MIP) são propriedades de campo que surgem da interação de uma sonda (por exemplo, metila, próton ou água) com uma molécula. Estas propriedades são calculadas no espaço ao redor da molécula.

Potenciais Eletrostáticos Moleculares (MEP, do inglês Molecular Electrostatic Potential) - Potenciais eletrostáticos moleculares (MEP) são propriedades eletrostáticas de uma molécula baseadas na densidade de carga calculada diretamente da função de onda molecular. O potencial eletrostático (escalar com dimensões de energia) é calculado em um ponto nas vizinhanças de uma molécula. A derivada espacial é a força elétrica (vetorial) que atua em uma carga unitária positiva naquele ponto causada pelos núcleos e elétrons da molécula ${ }^{31}$.

Potenciais Lipofílicos Moleculares (MLP, do inglês Molecular Lipophilic Potential) - Potenciais lipofílicos moleculares (MLP) são propriedades na superfície de Van der Waals ou na superfície molecular acessível ao solvente ou em qualquer outro ponto no espaço (por exemplo, na grade 3D para estudos CoMFA) calculadas a partir de contribuições de lipofilia atômicas. Pode ser usado para cálculos de log P, CoMFA e em estudos de ancoramento ${ }^{32}$.

Propriedades Principais - Propriedades principais são escalas de valores de substituintes ou de aminoácidos derivados da análise dos componentes principais de uma grande matriz de variáves descritoras de estrutura, úteis no planejamento em série e análise de dados.

QSAR-3D (do inglês Three-Dimensional Quantitative StructureActivity Relationships, Relações Quantitativas entre Estrutura Tridimensional e Atividade)* - Relações quantitativas entre estrutura tridimensional e atividade (QSAR-3D) envolvem a análise da relação quantitativa entre a atividade biológica de um conjunto de substâncias e suas propriedades tridimensionais por meio de métodos de correlação estatística.

Química Computacional* - Química computacional é uma disciplina que usa métodos matemáticos para o cálculo de propriedades moleculares e para a simulação do comportamento molecular. Também inclui, por exemplo, o planejamento de sínteses, a pesquisa de bancos de dados e a manipulação de bibliotecas combinatoriais ${ }^{33,34}$.

Química em Computador - Química em computador é frequientemente usado como equivalente de química computacional; pode também se referir ao uso de computadores no planejamento de sínteses ${ }^{34,35}$.

Quimiometria - Quimiometria é a aplicação da estatística na análise de dados químicos (de química orgânica, analítica ou medicinal) e no planejamento de experimentos e simulações em química.

Receptor* - Um receptor é uma proteína ou um complexo de proteínas localizado no interior ou na superfície de uma célula, que reconhece especificamente e interage com um composto ${ }^{q}$ que atua como um mensageiro molecular (neurotransmissor, hormônio, fármaco, etc). Em um sentido mais amplo, o termo receptor é freqüentemente usado como sinônimo para qualquer sítio específico de ligação de fármacos (em oposição a não específico, como a ligação às proteínas do plasma), também incluindo ácidos nucléicos, tais como o DNA. 
Reconhecimento de Padrões* - Reconhecimento de padrões é a identificação de padrões em grandes conjuntos de dados, usando uma metodologia matemática apropriada. Exemplos são a análise de componentes principais (PCA), SIMCA, mínimos quadrados parciais (PLS) e redes neurais artificias (ANN) $)^{6,36,37}$.

Redes Neurais - (veja Redes Neurais Artificiais)

Redes Neurais Artificiais - Redes neurais artificiais (ANN, do inglês artificial neural network) são algoritmos que simulam o funcionamento dos neurônios humanos e podem ser usadas em problemas de reconhecimento de padrões, para estabelecer, por exemplo, relações quantitativas estrutura-atividade.

Refratividade Molar (RM) - A refratividade molar é o volume molar corrigido pelo índice de refração. Esta representa o tamanho e a polarizabilidade de um fragmento ou de uma molécula.

Relações Quantitativas Estrutura-Atividade (QSAR*, do inglês Quantitative Structure-Activity Relationships) - Relações quantitativas estrutura-atividade (QSAR) são modelos matemáticos que relacionam a estrutura química e a atividade farmacológica de um modo quantitativo para uma série de compostos. Os métodos que podem ser usados em QSAR incluem várias técnicas de regressão e de reconhecimento de padrões.

QSAR é freqüentemente considerada como equivalente à quimiometria ou à análise estatística multivariada de dados. Às vezes é usada em um sentido mais limitado, como equivalente à análise de Hansch. QSAR é um subconjunto do termo mais geral SPC ${ }^{1}$.

SIMCA - O método SIMCA (do inglês SIMple Classification Analysis, Análise por Classificação Simples, ou Soft Independent Modeling of Class Analogy, Modelagem Independente Suave de Analogia de Classes) é uma técnica de reconhecimento de padrões e de classificação ${ }^{38}$.

SMILES - SMILES (do inglês Simplified Molecular Input Line Entry System, Sistema de Entrada de Linha de Dados Moleculares Simplificado) é uma notação em cadeia usada para descrever a natureza e a topologia de estruturas moleculares.

Superfície Acessível ao Solvente - A superfície acessível ao solvente é descrita como a superfície traçada por fora por uma molécula sonda, como por exemplo água, rolando sobre a superfície de Van der Waals de uma molécula. Há dois tipos: a) a superfície formada pelos pontos do centro de uma sonda esférica rolada ao redor da molécula no contato de Van der Waals e b) a superfície de contato (ou superfície de Connolly/Richards). (veja superfície de Connolly)

Superfície de Connolly - Superfície de Connolly é a envoltória traçada pelo ponto de contato de uma determinada sonda (uma esfera, por exemplo) e uma molécula de interesse, onde elas se tocam uma vez, acrescida da superfície de Van der Waals da sonda onde elas se tocam duas vezes ou mais (a superfície reentrante). É usada para visualizar a superfície molecular.

Técnica de Monte Carlo - A técnica de Monte Carlo é um procedimento de simulação que consiste em amostrar aleatoriamente o espaço conformacional de uma molécula.

Têmpera Simulada-Têmpera simulada é um procedimento usado em simulações de dinâmica molecular, no qual o sistema é deixado alcançar o equilíbrio a altas temperaturas e, então, resfriado lentamente para remover energia cinética e para permitir que trajetórias locais se ajustem em conformações locais de energia mínima.

Termos de Energia Não-ligados - Termos de energia não-ligados são funções de energia potencial que descrevem interações de Van der Waals, eletrostáticas e de ligação hidrogênio em um campo de força.
Topologia Molecular - Topologia molecular é a descrição do modo pelo qual os átomos estão ligados em uma molécula. (veja Índice topológico)

Valores CLOGP - Valores CLOGP são coeficientes de partição 1-octanol/água calculados, freqüentemente usados em estudos de correlação estrutura-propriedade ou de relação quantitativa estrutura-atividade (SPC/QSAR, do inglês Structure-Property Correlation e Quantitative Structure-Activity Relationship) ${ }^{39}$. (veja correlações estrutura-propriedade (SPC) e relação quantitativa estrutura-atividade (QSAR))

Variável Indicadora - Uma variável indicadora é um descritor que pode assumir apenas dois valores que indicam a presença $(=1)$ ou a ausência $(=0)$ de uma dada condição. É freqüentemente usada para indicar a ausência ou a presença de um substituinte ou de uma subestrutura. De forma mais abrangente, é uma variável que pode codificar tudo que um investigador escolhe.

\section{REFERÊNCIAS}

1. Kubinyi, H. Em Methods and Principles in Medicinal Chemistry; Mannhold, R. et al., eds.; VCH: Weinheim, 1993, vol. 1.

2. Rogers, D.; Hopfinger, A. J.; J. Chem. Inf. Comp. Sci. 1994, 34, 854.

3. Cramer III, R. D.; Patterson, D. E.; Bunce, J. D.; J. Am. Chem. Soc. 1988, $110,5959$.

4. Kubinyi, H.; 3D-QSAR in Drug Design. Theory, Methods and Applications, Escom: Leiden, 1993.

5. Hansch, C.; Fujita, T.; J. Am. Chem. Soc. 1964, 86, 1616.

6. Rouvray, D. H. Em Concepts and Applications of Molecular Similarity; Johnson, A. M.; Maggiora, G. M., eds.; Wiley: Nova Iorque, 1990.

7. Willet, P.; Similarity and Clustering in Chemical Information Systems, Wiley: Nova Iorque, 1987.

8. Willet, P.; Three-dimensional Chemical Structure Handling, Wiley: Nova Iorque, 1991.

9. Topliss, J. G.; J. Med. Chem. 1972, 15, 1006.

10. Wylie, W. A. Em Molecular Modeling and Drug Design; Vinter, J. G.; Gardner, M., eds.; Macmillan: Londres, 1994.

11. Bingham, R. C.; Dewar, M. J. S.; Lo, D. H.; J. Am. Chem. Soc. 1975, 97, 1285 .

12. Allinger, N. L.; J. Am. Chem. Soc. 1977, 99, 8127.

13. Hansch, C.; Leo, A.; Hoekman, D.; Exploring QSAR, American Chemical Society: Washington, 1995.

14. Rekker, R. F.; De Kort, H. M.; Eur. J. Med. Chem. 1979, 14, 479.

15. Hansch, C.; Leo, A.; Substituent Constants for Correlation Analysis in Chemistry and Biology, Wiley: Nova Iorque, 1979.

16. Rekker, R. F.; Mannhold, R.; Calculation of Drug Lipophilicity, VCH: Weinheim, 1992.

17. van de Waterbeemd, H.; Quant. Struct.-Act. Relat. 1992, 11, 200.

18. van de Waterbeemd, H.; Testa, B.; Adv. Drug Res. 1987, 16, 85.

19. Good, A. C.; J. Mol. Graph. 1992, 10, 144

20. Willet, P.; Winterman, V.; Quant. Struct.-Act. Relat. 1986, 5, 18.

21. Dalby, A.; Nourse, J. G.; Hounsell, W. D.; Gushurt, A. K. I.; Grier, D. L.; Leland, B. A.; Laufer, J.; J. Chem. Inf. Comput. Sci. 1992, 32, 244.

22. Crippen, G. M.; Havel, T. F.; Distance Geometry and Molecular Conformation, Wiley: Nova Iorque, 1988.

23. Goodford, P. J.; J. Med. Chem. 1985, 28, 849.

24. Martin, Y. C.; Quantitative Drug Design, Marcel Dekker: Nova Iorque, 1978.

25. Martin, Y. C. Em Modern Drug Research. Paths to Better and Safer Drugs; Martin, Y. C.; Austel, V.; Kutter, E., eds.; Marcel Dekker: Nova Iorque, 1989, p. 161-216.

26. Dean, P. M. Em Concepts and Applications of Molecular Similarity; Johnson, A. M.; Maggiora, G. M., eds.; Wiley: Nova Iorque, 1990, p. 211.

27. Kier, L. B.; Hall, L. H.; Molecular Connectivity in Chemistry and Drug Research, Academic Press: Londres, 1976.

28. Burkert, U.; Allinger, N. L.; Molecular Mechanics, ACS Monograph 177, 1982 .

${ }^{\mathrm{q}} N$. T.: Na maior parte dos casos, o processo de interação é constituído de uma soma de interações intermoleculares fracas entre o ligante e o receptor. O processo de interação possui também uma significativa contribuição entrópica relacionada, por exemplo, com mudanças na rotação, na translação e na vibração das moléculas inteiras envolvidas (inclusive as do solvente) e de seus fragmentos. 
29. Wold, S.; Johansson, E.; Cocchi, M. Em 3D-QSAR in Drug Design. Theory, Methods and Applications; Kubinyi, H., ed.; Escom: Leiden, 1993, p. 523.

30. Verloop, A.; The STERIMOL Approach to Drug Design, Marcel Dekker: Nova Iorque, 1987.

31. Williams, D. E.; Rev. Comp. Chem. 1991, 2, 226.

32. Gaillard, P.; Carrupt, P. M.; Testa, B.; Boudon, A.; J. Comp. Aided Mol. Des. 1994, 8, 83 .

33. Hopfinger, A. J.; J. Med. Chem. 1981, 24, 119.

34. Ugi, I.; Wochner, M.; Fontain, E.; Bauer, J.; Gruber, B.; Karl, R. Em Concepts and Applications of Molecular Similarity; Johnson, A. M.; Maggiora, G. M., eds.; Wiley: Nova Iorque, 1990, p. 239.
35. Boyd, D. B., ed.; Reviews in Computational Chemistry, 1990, vol. 1.

36. van de Waterbeemd, H. Em Methods and Principles in Medicinal Chemistry; Mannhold, R. et al., eds.; VCH: Weinheim, 1995, vol. 2.

37. van de Waterbeemd, H. Em Methods and Principles in Medicinal Chemistry; Mannhold, R. et al., eds.; VCH: Weinheim, 1995, vol. 3.

38. Dunn, W. J.; Wold, S. Em Chemometric Methods in Molecular Design; van de Waterbeemd, H., ed,; VCH: Weinheim, 1995, p. 179.

39. Leo, A. J.; Chem. Revs. 1993, 93, 1281. 